\title{
CONHECIMENTO E MODIFICAÇÕES DE COMPORTAMENTO FRENTE AO TRATAMENTO NÃO-FARMACOLÓGICO DAHAS: ANTES E APÓS EDUCAÇÃO EM SAÚDE DO PROFISSIONAL ENFERMEIRO
}

\author{
Danusa Fernandes Severo ${ }^{1}$, Simone Coelho Amestoy², Maira Buss Thofehrn ${ }^{3}$, Silvia Goldmeier ${ }^{4}$
}

\begin{abstract}
RESUMO: Objetivo: verificar o conhecimento sobre o tratamento não-farmacológico da Hipertensão Arterial SistêmicaHAS e as modificações de comportamento frente a este tipo de tratamento, antes e após educação em saúde do profissional enfermeiro, em pacientes hipertensos submetido à hemodiálise. Pesquisa-ação, com abordagem quantitativa. Foi dividido em três etapas: coleta de dados, educação em saúde, coleta de dados. Totalizaram 19 pacientes com hipertensão primária submetidos à sessões de hemodiálise em uma clínica renal de Torres-RS, de outubro a dezembro de 2007. Verificou-se que a Educação em Saúde pelo profissional enfermeiro com os pacientes hipertensos submetidos à hemodiálise foi positiva, visto que contribuiu na ampliação do conhecimento dos pacientes frente ao seu tratamento e, embora sutis, também nas mudanças no comportamento frente ao tratamento não-farmacológico da HAS, contribuindo na promoção de saúde dos pacientes do estudo para o autocuidado, contemplando os objetivos da Educação em Saúde.
\end{abstract}

PALAVRAS-CHAVE: Enfermagem; Educação em saúde; Hipertensão.

\section{KNOWLEDGE AND CHANGES IN BEHAVIOR REGARDING THE NON- PHARMACOLOGICAL TREATMENT OF THE SAH: BEFORE AND AFTER HEALTH EDUCATION PERFORMED BY A NURSE}

\begin{abstract}
Objective: to verify the knowledge about the non-pharmacological treatment of the SAH and the changes in behavior regarding this type of treatment, before and after health education performed by a nurse, with patients with the hypertension diagnose that were submitted to hemodialysis. This was an exploratory and descriptive research, an action research, of quantitative approach. It was divided in three stages: data collection, education in health, data gathering. Subjects were 19 patient with primary hypertension submitted to hemodyalisis sessions in a renal clinic of Torres-RS, from October to December 2007. It was verified that the Education in Health performed by the nurse with hypertensive patients submitted to the hemodyalisis was positive, because it contributed to grant more knowledge to the patients about their treatment and also to behavioral changes regarding non-pharmacological treatment of the SAH, contributing to these patient's health promotion and also to their selfcare, contemplating the objectives of the Education in Health.
\end{abstract}

KEYWORDS: Nursing; Health education; Hypertension.

\section{CONOCIMIENTO Y MODIFICACIONES DE COMPORTAMIENTO FRENTE AL TRATAMIENTO NO FARMACOLÓGICO DE LA HAS: ANTES Y DESPUÉS DE LA EDUCACIÓN EN SALUD DEL PROFESIONAL ENFERMERO}

\begin{abstract}
RESUMEN: Objetivo: verificar el conocimiento acerca del tratamiento no farmacológico de la Hipertensión Arterial Sistémica - HAS y las modificaciones de comportamiento frente a este tipo de tratamiento, antes y después de la educación en salud del profesional enfermero, en pacientes hipertensos sometidos a la hemodiálisis. Investigación-acción, con abordaje cuantitativo. Fue dividido en tres etapas: colecta de datos, educación en salud, recopilación de datos. Totalizaron 19 pacientes con hipertensión primaria sometidos a sesiones de hemodiálisis en una clínica renal de Torres-RS, de octubre a diciembre de 2007. Fue verificado que la Educación en Salud por el profesional enfermero con los pacientes hipertensos sometidos a hemodiálisis fue positiva, visto que contribuyó para la ampliación del conocimiento de los pacientes frente a su tratamiento y, aunque sutiles, también los cambios en el comportamiento frente al tratamiento no farmacológico de la HAS, contribuyendo para la promoción de la salud de los pacientes del estudio para el autocuidado, contemplando los objetivos de la Educación en Salud.
\end{abstract}

PALABRAS CLAVE: Enfermería; Educación en salud; Hipertensión.

${ }^{1}$ Enfermeira. Especialista em Cardiologia pelo Programa de Pós-graduação da Fundação Universitária de Cardiologia do Rio Grande do Sul-FUC-RS.

${ }^{2}$ Enfermeira. Mestre em Enfermagem pela Fundação Universidade Federal do Rio Grande-FURG. Especialista em Enfermagem com ênfase em Terapia Intensiva pela Associação Hospitalar Moinhos de Vento-Poa.

${ }^{3}$ Enfermeira. Doutora em Enfermagem. Professora da Universidade Federal de Pelotas-UFPel-RS.

${ }^{4}$ Enfermeira. Mestre em Enfermagem pelo Curso de Pós-graduação de Enfermagem em Cardiologia do Rio Grande do Sul.

Autor correspondente:

Danusa Fernandes Severo

Rua Felix Cunha, 08/309 - 9601-000 - Pelotas-RS

Recebido: 03/08/08

E-mail: danusa.enf@hotmail.com

Aprovado: 10/08/09

Cogitare Enferm 2009 Jul/Set; 14(3):506-11 


\section{INTRODUÇÃO}

A Hipertensão Arterial Sistêmica-HAS é um grande fator de risco para desenvolvimento de Doença Arterial Coronariana-DAC, Acidente Vascular CerebralAVC, Doença Valvar Periférica, Insuficiência Renal Crônica-IRC e Insuficiência Cardíaca Congestiva-ICC, sendo uma das mais importantes causas de morbimortalidade universal ${ }^{(1-2)}$.

A HAS também é responsável pela grande maioria das nefropatias, das lesões extra-renais e cerca de $10 \%$ dos óbitos dos pacientes hipertensos estão relacionados à IRC. Quando a função renal diminui de 10 a $12 \%$, as terapias dialíticas substitutivas são indicadas. Segundo o censo da Sociedade Brasileira de Nefrologia de 2006, há 64.306 pacientes submetidos à hemodiálise, 6.566 submetidos a diálise peritoneal, e cerca de 60 a $80 \%$ da população dialítica são hipertensos ${ }^{(2-3)}$. Nesses pacientes, quanto menor 0 controle da Hipertensão Arterial maiores são as taxas de mortalidade, tanto no primeiro ano, quanto nos anos subsequentes de tratamento dialítico ${ }^{(4)}$. Todavia, o controle da Pressão Arterial-PA melhora o prognóstico do indivíduo, diminuindo assim a mortalidade desses ${ }^{(3)}$.

A pressão arterial pode ser controlada através de tratamento não-farmacológico da HAS, no qual se inclui a realização de atividade física, restrição de alimentos ricos em sódio, moderação do consumo de bebidas alcoólicas, abandono do tabagismo, controle do peso e do estresse psicoemocional ${ }^{(2,5-6)}$. A educação em saúde tem contribuído significativamente para a prevenção e controle de doenças nos últimos 20 anos, e objetiva fornecer conhecimento ao paciente com a finalidade de estimulá-lo para efetivar mudanças em seu comportamento ${ }^{(7)}$.

É possível constituir motivos relevantes para uma atuação eficaz do enfermeiro quanto à condução do paciente hipertenso ao tratamento adequado da hipertensão arterial. A efetividade da participação da enfermeira de maneira sistemática está possivelmente fundamentada em sua atuação no aspecto psicoemocional do paciente, também na orientação e elucidação de dúvidas sobre a doença, tratamento e fatores de risco; e em instruções sobre aspectos de higiene, hábitos alimentares, crenças de saúde ${ }^{(8)}$.

Para realizar a Promoção da saúde através de estratégias educativas em saúde, principalmente numa base dialógica, a enfermagem respeita os preceitos éticos e legais internacionais, constitucionais e de sua profissão, assim como, o indivíduo na sua dignidade, liberdade e autonomia ${ }^{(9)}$. Perante os descritos, é de grande importância a atuação do enfermeiro na educação em saúde, priorizando as atividades inerentes ao seu papel de educador, que oportuniza o engajamento do paciente nas atividades de autocuidado, principalmente quando apresenta déficit de conhecimento e de habilidade para cuidar de si mesmo.

Em estudo que avaliou a eficácia de um programa de pré e pós-consultas intermediado por enfermeiras, capacitadas para educação em saúde quanto ao controle da hipertensão arterial, constatou-se que $23 \%$ dos pacientes mantiveram a PA controlada, enquanto no grupo com atendimento convencional este índice se manteve em $12 \%^{(10)}$. Em outro estudo, realizado com 353 pacientes hipertensos atendidos no mesmo hospital do anterior, foi possível identificar que $81 \%$ desconheciam o tratamento não-farmacológico ${ }^{(11)}$. O estudo transversal sobre a adesão ao tratamento no grupo de hipertensos do bairro Joaquim Romão-JequiéBA, envolvendo 35 pacientes, em janeiro e fevereiro de 2006 , verificou que $63,8 \%$ admitiram seguir regularmente o tratamento. Destes, apenas 27,8\% faziam uso do tratamento não-medicamentoso ${ }^{(12)}$.

Conquanto, no presente estudo salienta-se a importância do enfermeiro como educador em saúde, proporcionando aos pacientes hipertensos o conhecimento sobre sua doença, seu tratamento nãofarmacológico. Visando, desta forma, a prevenção e progressão do desenvolvimento das patologias referidas, possibilitando modificações positivas no estilo de vida, promovendo o autocuidado do paciente. $\mathrm{O}$ interesse pelo tema emergiu no decorrer da atuação em enfermagem, ao se perceber que os pacientes hipertensos submetidos às sessões de hemodiálise pouco aderiam ao tratamento não-farmacológico da HAS.

Diante dos descritos, o estudo foi realizado com o objetivo de verificar o conhecimento sobre o tratamento não-farmacológico da HAS e as modificações de comportamento frente a este tipo de tratamento, antes e após educação em saúde do profissional enfermeiro, em pacientes hipertensos submetido à hemodiálise.

\section{MÉTODOS}

O delineamento do estudo foi do tipo descritivo exploratório, pesquisa-ação, com abordagem quantitativa. A pesquisa ação é caracterizada pelo envolvimento dos pesquisados e participantes representativos da situação ou do problema de modo 
cooperativo ou participativo ${ }^{(13)}$.

Realizado em uma clínica renal terceirizada, cidade de Torres-RS, no período de outubro a dezembro 2007. Nessa época, a clínica atendia 43 pacientes submetidos à hemodiálise. Dispunha de 10 máquinas, sendo uma destas exclusivamente para pacientes portadores de hepatite $\mathrm{B}$; e, duas para pacientes portadores de hepatite $\mathrm{C}$.

Os sujeitos preencheram os seguintes critérios de inclusão: pacientes com hipertensão arterial essencial, maiores de 18 anos, aceitar participar no estudo assinando o Termo de Consentimento Livre e Esclarecido-TCLE. Desse modo, totalizaram 19 pacientes hipertensos que se submeteram às sessões de hemodiálise, naquele período.

O estudo foi aprovado pela Comissão de Ética e Pesquisa do Instituto de Cardiologia do Rio Grande do Sul-Fundação Universitária sob. o no 4037/07 e autorizado pela responsável da instituição onde o mesmo foi realizado. Todos os pacientes concordaram em assinar o Termo de Consentimento Livre e Esclarecido.

O estudo foi dividido nas etapas: coleta de dados, educação em saúde e coleta de dados.

$1^{\text {a }}$ etapa - Coleta de Dados: Após a assinatura do TCLE, em duas vias, os pacientes foram convidados a responder um questionário distribuído em duas categorias. A primeira continha variáveis relacionadas às características demográficas e clínicas: sexo, idade, etnia, estado civil, anos de estudo, profissão, situação familiar, e tempo de HAS e de hemodiálise. Já na segunda, as variáveis referiam-se aos conhecimentos do tratamento não-farmacológico da HAS e às modificações de comportamento frente a esse tipo de tratamento.

$2^{\mathrm{a}}$ etapa - Educação em Saúde: Realizou-se educação em saúde do profissional enfermeiro constituído de abordagem individual com os sujeitos do estudo. Utilizou-se como ferramenta um folder informativo abordando o conceito, fisiopatologia básica da HAS e o tratamento não-farmacológico da HAS. Primeiramente ocorreu a distribuição do folder para o paciente e a explicação do conteúdo de modo dialógico, orientando-o, com o intuito de conduzir o paciente para o autocuidado. Dessa maneira, uma vez por semana, foram realizados encontros.

$3^{\text {a }}$ etapa - Coleta de Dados: Transcorridos 30 dias da entrega do folder, o mesmo questionário foi aplicado para análise das eventuais modificações ocorridas, da mesma forma a PA foi verificada como medida de parâmetro e o Peso Seco coletado do prontuário.
Na análise dos dados as variáveis contínuas foram descritas através de médias e desvios-padrão. Já, as variáveis categóricas foram descritas através de proporções e utilizado o programa SPSS 15. Os resultados foram expressos através de gráficos e tabelas construídos a partir do programa Excel do pacote Office 2007.

\section{RESULTADOS}

\section{Características clínicas e demográficas}

Participaram do estudo 19 pacientes, com idade $55 \pm 18,58 \%$ do sexo masculino, $89 \%$ de etnia branco, $53 \%$ casados e 5,35 $\pm 3,6$ anos de estudo. O tempo de HAS e hemodiálise foram $14,85 \pm 12$ anos e 3,81 \pm 4 meses, respectivamente. Informações adicionais à história clínica dos pacientes demonstraram que 32\% apresentaram cardiopatia isquêmica e 21\% Diabetes Mellitus-DM.

Tabela 1 - Características demográficas e clínicas da amostra

\begin{tabular}{lc}
\hline Características demográficas e clínicas & N. (\%) \\
\hline Sexo & $11(58)$ \\
Masculino & $08(42)$ \\
Feminino & $55 \pm 18$ \\
Idade Média* & \\
Etnia & $17(89)$ \\
Branca & \\
Estado Civil & $04(21)$ \\
Solteiro & $10(53)$ \\
Casado & $02(10)$ \\
Desquitado & $03(16)$ \\
Viúvo & $5,35 \pm 3,6$ \\
Estudo (anos)* & \\
Profissão & $06(32)$ \\
Agropecuária & $06(32)$ \\
Do lar & $02(10)$ \\
Técnica & $05(26)$ \\
Construção Civil & \\
Situação familiar & $16(84)$ \\
Com familiares & $3,81 \pm 4$ \\
Tempo de hemodiálise (anos)* & $14,85 \pm 12$ \\
Tempo de HAS (anos)* & \\
*Média $\pm:$ desvio-padrão. &
\end{tabular}


Conhecimento sobre o tratamento nãofarmacológico da HAS: antes e após educação em saúde

Pode-se visualizar na figura 1 o entendimento informado pelos pacientes no momento da aplicação do questionário sobre o tratamento não-farmacológico da HAS, com a subsequente educação em saúde do enfermeiro e, 30 dias após, cujo questionário foi novamente aplicado. No item atividade física houve um acréscimo de conhecimento de 16 para 68\%; no controle do sal o acréscimo foi de 89 para 95\%, abandono à ingestão de bebidas alcoólicas o percentual teve o acréscimo de 5 para 11\%. No item de abandono ao tabagismo o percentual aumentou de 0 para $16 \%$; e houve um aumento de 5 para $16 \%$ do conhecimento sobre a importância da redução do peso como um dos tratamentos não-farmacológico da HAS.

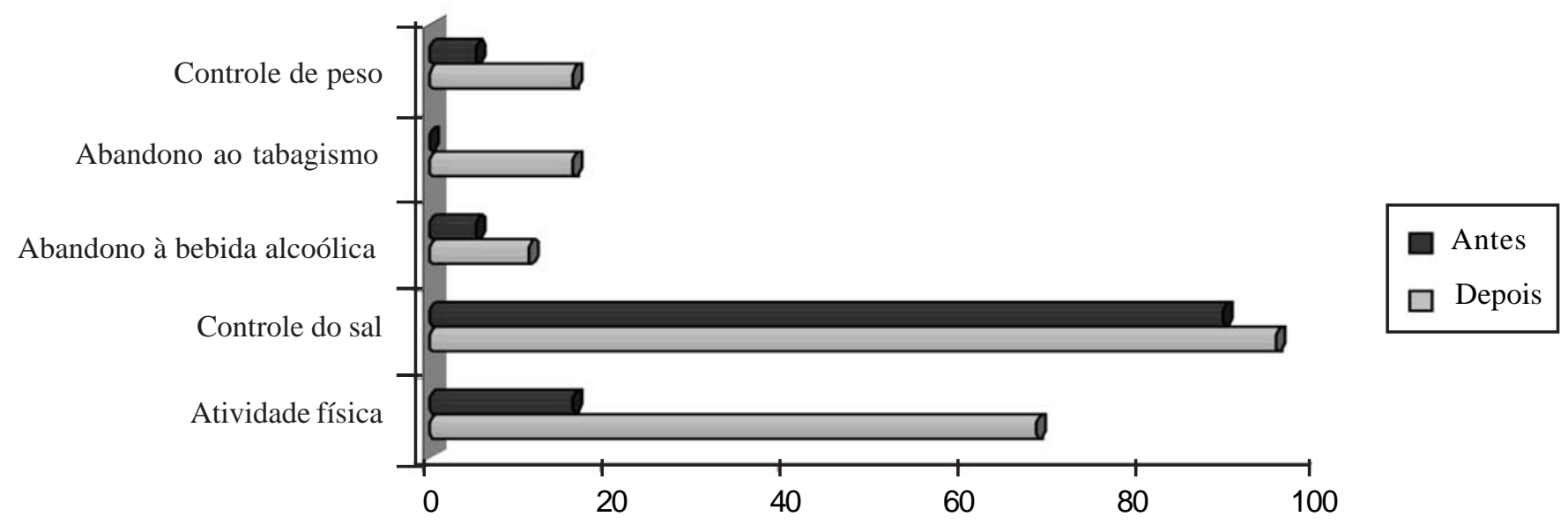

Figura 1 - Conhecimento sobre o tratamento não-farmacológico da HAS: antes e após educação em saúde

Legenda do eixo y: Tratamento não-farmacológico da HAS

Legenda do eixo x: Conhecimento dos pacientes hipertensos submetidos à hemodiálise sobre o tratamento não-farmacológico da HAS, antes e após Educação em Saúde do Profissional enfermeiro (\%)

Modificação de comportamento após a educação em saúde

Na figura 02 está demonstrada a adesão do tratamento não-farmacológico da HAS, do paciente hipertenso submetido à hemodiálise, antes e após Educação em Saúde. A realização de atividade física aumentou de $53 \%$ para $84 \%$, o controle da ingestão de sal manteve-se em 58\%, a ingestão de bebida alcoólica reduziu em $6 \%$, hábito de fumar reduziu em $5 \%$.

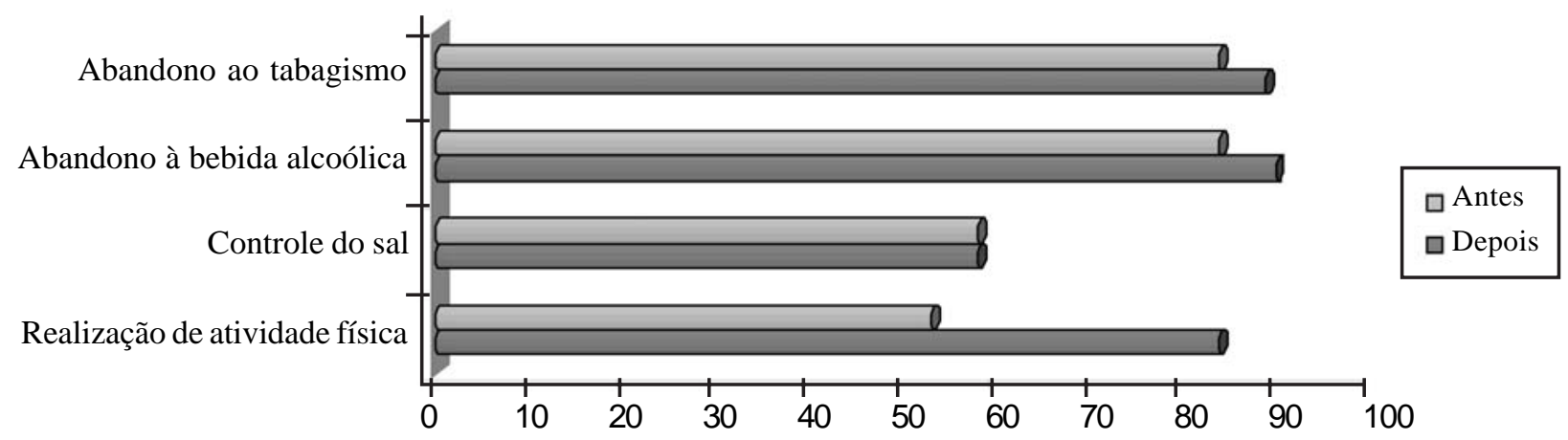

Figura 02 - Adesão do tratamento não-farmacológico da HAS, do paciente hipertenso submetido à hemodiálise, antes e após educação em saúde

Legenda do eixo y: Tratamento não-farmacológico da HAS

Legenda do eixo x: Adesão ao tratamento não-farmacológico da HAS antes e após a Educação em Saúde pelo profissional enfermeiro (\%) 


\section{DISCUSSÃO}

No presente estudo, após a realização da educação em saúde pelo enfermeiro é notório uma melhora do conhecimento dos pacientes hipertensos submetidos à hemodiálise quanto ao tratamento nãofarmacológico da HAS, além de um pequeno percentual de modificações de comportamento quanto à adesão ao tratamento. Os pacientes com IRC e HAS deparam-se constantemente com limitações em seu cotidiano, pois o tratamento é complexo, motivo pelo qual se faz necessário estimular sua capacidade de adesão a um novo modo de viver e, também, de assumir o controle de seu tratamento a partir do conhecimento adquirido $^{(15)}$.

Em relação ao conhecimento sobre o tratamento não-farmacológico da HAS podemos observar que tivemos como item mais conhecido o controle da ingesta de sal, tanto antes quanto depois da Educação em Saúde. Esse achado coaduna com o estudo realizado com 353 pacientes hipertensos, sobre o conhecimento do tratamento não-farmacológico e farmacológico da HAS, no qual 96\% dos pacientes hipertensos tinham como item mais conhecido a redução da ingesta de $\mathrm{sal}^{(11)}$.

Quanto às modificações de comportamento, o tratamento não-farmacológico com maior percentagem foi a realização de atividade física. A combinação de atividade física e de uma dieta adequada tem como resultado, além de outros benefícios, a redução dos níveis pressóricos ${ }^{(15)}$. A recomendação para este tratamento tem como parâmetros a frequência, mantida em cinco vezes por semana com duração mínima de 30 minutos e intensidade moderada ${ }^{(3)}$. A realização da atividade física regularmente necessita um período entre 2 a 6 meses para se obter um efeito hipotensor ${ }^{(17)}$. Um estudo de revisão de literatura verificou que em pacientes hipertensos a redução máxima da PA sistólica e diastólica após o exercício é entre 18 a $20 \mathrm{mmHg}$ e de 7 a $9 \mathrm{mmHg}$ respectivamente; já nos indivíduos normotensos esta redução é entre 8 a $10 \mathrm{mmHg}$ e de 3 a $5 \mathrm{mmHg}$, demonstrando que, quanto maior o nível pressórico inicial da PA em repouso, maior será a queda após exercício ${ }^{(18)}$.

É possível observar através dos resultados a manutenção da porcentagem dos pacientes que realizaram um controle na ingestão de sal. Estudos observacionais verificaram que a redução do consumo de sal tem implicações favoráveis sobre o controle pressórico. Um estudo de 2,5 anos, com uma amostra de 1.131 pacientes hipertensos que foram divididos em quatro grupos (restrição de sódio, redução de sal e peso, grupo controle) verificou a redução de PA em $38 \%$ do grupo com restrição de sódio ${ }^{(19)}$.

Quanto ao uso de bebida alcoólica, após 30 dias houve redução no número de pacientes que faziam uso. O aumento da PA está associada à quantidade de etanol ingerida e à frequência desta ingestão. A redução média pode atingir níveis de 3,3 mmHg na PA sistólica e 2,0 mmHg na diastólica, com a redução de ingestão de etanol $^{(2)}$. Um estudo longitudinal realizado com uma amostra de 970 pacientes mostrou um aumento da PA nos indivíduos que iniciaram o consumo de álcool durante o estudo, sendo que a PA dos pacientes que deixaram de beber durante o estudo retornou aos níveis dos indivíduos que não ingeriam bebida alcoólica( ${ }^{(16)}$.

A nicotina do cigarro libera a catecolamina, que promove um aumento agudo da PA e cuja liberação é proporcional ao número de cigarros fumados agregado à profundidade da inalação ${ }^{(19)}$. O hábito de fumar foi abandonado por uma pequena parcela da amostra.

\section{CONSIDERAÇÕES FINAIS}

Diante dos resultados apresentados e discutidos, a Educação em Saúde do profissional enfermeiro aos pacientes hipertensos submetidos à hemodiálise foi positiva, visto que contribuiu na ampliação do conhecimento dos pacientes frente ao seu tratamento e, embora sutis, também nas mudanças no comportamento frente ao tratamento nãofarmacológico da HAS, contribuindo na promoção de saúde dos pacientes do estudo para o autocuidado, contemplando os objetivos da Educação em Saúde.

Assim, pode-se dizer que adquirir conhecimento sobre hipertensão é uma das estratégias para a modificação de comportamento, adesão ao tratamento não-farmacológico da HAS, e subsequente adoção de modificação no estilo de vida, autocuidado do paciente. Convém destacar que apenas disponibilizar informações não garantirá o comprometimento dos pacientes às mudanças necessárias: é preciso usar as informações através de um diálogo, cujo estímulo deverá ser constante e individual a cada paciente.

É nesse contexto que o profissional enfermeiro tem o compromisso com o processo de educação em saúde, o qual se tornará mais efetivo se forem aplicados os conhecimentos teóricos em sua prática educativa, considerando os aspectos histórico-sócio-culturais do paciente. 


\section{REFERÊNCIAS}

1. Andrade J, Vilas-Boas F, Chagas H, Andrade M. Aspectos epidemiológicos da aderência ao tratamento da hipertensão arterial sistêmica. Arq Bras Cardiol. 2002 Out;79(4):375-79.

2. V Diretrizes Brasileiras de Hipertensão Arterial. 2006. [Acesso em 2007 Ago 20] Disponível: http://www.sbn. org.br/Diretrizes/V_Diretrizes_Brasileiras_de_Hiperten sao_Arterial.pdf.

3. Ministério da Saúde (BR). Secretaria de Atenção à Saúde. Secretaria de Atenção à Saúde. Departamento de Atenção Básica. Caderno de Atenção Básica Hipertensão Arterial Sistêmica. nº.15. Brasília; 2006.

4. Oliveira JMF. Revisão/atualização em diálise: hipertensão arterial em hemodialisados - fatores relacionados ao seu controle adequado ou inadequado e tratamento. J Bras Nefrol.1997;19(2):212-14.

5. Brunner e Suddarth. Tratado de enfermagem médicocirúrgico. 9a ed. Rio de Janeiro: Guanabara Koogan; 2002.

6. Ministério da Saúde (BR). Plano de Reorganização da Atenção à Hipertensão Arterial e ao Diabetes Mellitus. Brasilia 2002. [Acesso em 2008 Jun 10]. Disponível: http:/ /dtr2004.saude.gov.br/dab/hipertensaodiabetes/ publicacoes.php

7. Zernike WBN, Henderson ABS. Evaluating tehe effectiveness of two teching strategies for patients diagnosed with hypertension. J Clin Nurs 1998;7:37-44.

8. Pierin AMG, Car MR, Giorgi DMA, Mion JD. Atendimento de enfermagem ao paciente com hipertensão arterial. Rev Bras Med Cardiol. 1984Ago;3(4):209-11.

9. Shiratori K, Costa TL, Formozo GA, Silva AS. Educação em saúde como estratégia para garantir a dignidade da pessoa humana. Rev Bras Enferm. 2004 Set/ Out;57(5):617-19.

10. Giorgi DMA. Estratégias para melhorar a adesão ao tratamento anti-hipertensivo. Rev Bras Hipert. 2006 Jan/ Mar;13(1):47-50.

11. Mion JDM, Pierin A, Ignez E, Ballas D, Marcondes M. Conhecimento, preferências e perfil dos hipertensos quanto ao tratamento farmacológico e não farmacológico. J Bras Nefrol. 1995;17(4):229-36.

12. Meira CH, Mascarenhas, Oliveira MML, Souza MS.
Adesão ao tratamento no grupo de hipertensos do bairro Joaquim Romão-Jequié/BA. Rev Saúde Com. 2006 Jan/Mar;2(1):30-8.

13. Silva EL, Menezes EM. Metodologia da pesquisa e elaboração de dissertação. Rev Atual. Florianópolis: Laboratório de Ensino a Distância da Universidade Federal de Santa Catarina/ SC. 2001. $3^{\mathrm{a} e d .121 p .}$

14. Mendes RB, Santos SFF. Hipertensão Arterial na Hemodiálise [periódico na Internet]. Rev Virtual de Medicina. 2000 [Acesso em 2008 Jun 11]. 9(1). Disponível: http://www.medonline.com.br/med_ed/ med9/hipehem.htm.

15. Cesarino CB, Casagrande LDR. Paciente com insuficiência renal crônica em tratamento hemodialitico: atividade educativa do enfermeiro. Rev Latino-Am Enferm. 1998 Out;6(4):31-40.

16. Fajardo C. A importância da abordagem nãofarmacológica da hipertensão arterial na atenção primaria à saúde. Rev Bras Med Fam e Com. 2006 Jan/ Mar;4(1):107-18.

17. Forjaz CL, Santaella DF, Souza MO. A atividade física e a pessoa hipertensa. In: Pierin AM. Hipertensão arterial: um proposta para o cuidar. Barueri: Manole; 2004. p185204.

18. Laterza MC, Rondon MU, Negrão CE. Efeito antihipertensivo do exercício. Rev Bras Hipert. 2007 Abr/ Jun;14(2):104-11.

19. Borges JL, Gravina CF, Grespan SM. Tratamento nãomedicamentoso da hipertensão no Idoso. Rev Bras Hipert. 2007 Jan/Mar;14(1):33-6. 